\title{
PRIMITIVE IDEALS IN GROUP RINGS OF POLYCYCLIC GROUPS
}

\author{
ROBERT L. SNIDER
}

\begin{abstract}
If $F$ is a field which is not algebraic over a finite field and $G$ is a polycyclic group, then all primitive ideals of the group ring $F[G]$ are maximal if and only if $G$ is nilpotent-by-finite.
\end{abstract}

We recall that a primitive ring is a ring with a faithful irreducible module. An ideal is primitive if the factor ring is a primitive ring.

If $F$ is a field algebraic over a finite field and $G$ a polycyclic group, then Roseblade has recently shown that every irreducible module for the group ring $F[G]$ is finite dimensional [5]. This implies that the primitive factor rings are simple Artin. On the other hand, if $F$ is any other field and $G$ is not abelianby-finite, then Hall observed that $F[G]$ has infinite dimensional irreducible modules [2]. If $G$ is finitely generated nilpotent, Zalesskii proved that the primitive factor rings are at least simple for any field $F[6]$. In this paper we offer a converse to Zalesskiirs theorem by proving the

THEOREM. If $F$ is a field which is not algebraic over a finite field and $G$ is a polycyclic group, then all primitive ideals of the group ring $F[G]$ are maximal if and only if $G$ is nilpotent-by-finite.

We warn the prospective reader of Zalesskii's paper [6] that the word "primitive" has been translated as prime throughout.

LEMMA 1 . If $G$ is polycyclic and $H$ is a subgroup of finite index with all primitive ideals of $F[H]$ maximal, then all primitive ideals of $F[G]$ are also maximal.

Proof. The proof of Theorem 3 in [6] applies.

Lemma 2. Let $G$ be polycyclic and $H$ a subgroup of finite index in $G$. If $F[H]$ has a nonmaximal primitive ideal, then $F[G]$ does also.

Proof. By Lemma 1, we may assume $H$ is normal in $G$. Let $P$ be a primitive ideal of $F[H]$ properly contained in a maximal ideal $Q$. Let $1=g_{1}, g_{2}, \ldots, g_{n}$ be a set of coset representatives for $H$ in $G$. Let $\bar{P}=\cap_{i=1}^{n} g_{i}^{-1} P g_{i}$ and $\bar{Q}$ $=\cap_{i=1}^{n} g_{i}^{-1} Q g_{i} . \bar{P} \varsubsetneqq \bar{Q}$ since equality would imply that $P \supseteq g_{i} Q g_{i}^{-1}$ for some $i$ and hence $P$ would be maximal. Let $V$ be an irreducible $F[H]$ module with annihilator $P . \bar{V}=V \otimes_{F[H]} F[G]$ has finite length as an $F[H]$ module and hence as an $F[G]$ module. Let $0=W_{0} \subseteq W_{1} \subseteq \cdots \subseteq W_{n}=\bar{V}$ be a $F[G]$ composition series for $\bar{V}$. The annihilator of $\bar{V}$ is $\bar{P} G$. $\bar{Q} G$ is a two-sided ideal

Received by the editors March 7, 1975.

AMS (MOS) subject classifications (1970). Primary 16A26. 
and hence can be embedded in a maximal ideal $M$. Let $P_{i}=\operatorname{Ann}\left(W_{i} / W_{i-1}\right)$. Since $P_{n} P_{n-1} \cdots P_{1}$ annihilates $\bar{V}, P_{n} P_{n-1} \cdots P_{1} \subseteq \bar{P} G \subseteq \bar{Q} G \subseteq M$. Hence $M$ contains $P_{i}$ for some $i$. Now $W_{i} / W_{i-1}$ contains a copy of $V g_{j}$ for some $j$ and hence contains a copy of $V g_{j}$ for each $j$. Hence $P_{i} \cap F[H]$ $=\operatorname{Ann}_{F[H]} W_{i} / W_{i-1}=\bigcap_{i=1}^{n}$ Ann $\left(V g_{i}\right)=\bar{P}$. Since $M \cap F[H] \supseteq \bar{Q} G$ $\cap F[H]=\bar{Q}$, we have $P_{i} \varsubsetneqq M$ and $P_{i}$ is a nonmaximal primitive ideal.

Proof of THEOREM. If $G$ is nilpotent-by-finite, the result follows from Zalesskii [6] and Lemma 1 . Conversely suppose $G$ is not nilpotent-by-finite. Pick a subgroup $K$ maximal among the subgroups with $N=\Re_{G}(K)$ of finite index and $N / K$ not nilpotent-by-finite. Using Lemma 2, we may assume $K$ is normal. Also since $F[G / K]$ is a homomorphic image of $F[G]$ we assume $K=1$. The finite conjugate subgroup of $G$ is trivial. Otherwise $G$ has a nontrivial normal subgroup $H$ whose centralizer $C$ has finite index. $C / C \cap H$ $\cong \mathrm{CH} / \mathrm{H}$ is nilpotent-by-finite by the maximality of $K$. Also $C \cap H$ is central in $C$ and hence $C$ and therefore $G$ is nilpotent-by-finite, a contradiction. Let $A$ be a maximal abelian normal subgroup. $G$ contains a subgroup $G_{1}$ of finite index such that $A$ contains a normal subgroup $B$ of $G_{1}$ with the property that $G_{1}$ and all of its subgroups of finite index act rationally irreducibly on $B$ [5, Lemma 2]. By Lemma 2, we assume $G=G_{1} . A$ is torsion free since $G$ has trivial finite conjugate subgroup. The rank of $B$ is at least two for the same reason. We may clearly replace $B$ with $Q B \cap A . Q A$ is a $Q[G / A]$ module and $Q B$ is an irreducible submodule. We claim that $Q A$ is an essential extension of $Q B$. Suppose to the contrary that $T$ is a nontrivial $Q[G / A]$ submodule with $T \cap Q B=0 . T \cap A$ is a nontrivial normal subgroup of $G$ and $G /(T \cap A)$ is nilpotent-by-finite. This is impossible since $G / T \cap A$ contains an isomorphic copy of $B$ and the rank of $B$ is at least two. Again using Lemma 2, we may assume $G / B$ is nilpotent. Let $U / Q B$ be an irreducible $Q[G / A]$ submodule of $Q A / Q B . U \cap A / B$ must intersect the center of $G / B$ nontrivially. Therefore, $U / Q B$ must have $Q$ dimension 1. Clearly Ann $(U) \subseteq$ Ann $(Q B)$. If $P_{1}$ $=$ Ann $U / Q B$ and $P_{2}=$ Ann $Q B$, then $P_{1} \neq P_{2}$ since $Q[G / A] / P_{1}$ $\cong Q$ and $Q[G / A] / P_{2}$ has dimension greater than 1. If Ann $U=$ Ann $Q B$, then $P_{2} \subseteq P_{1}$, but this is impossible since each has cofinite dimension and hence are maximal. Therefore, Ann $(U) \subsetneq \operatorname{Ann}(Q B)$. By [4], there is an $x \neq 0$ in the center of $Q[G / A] / \operatorname{Ann}(U)$ with $x$ in Ann $(Q B) / \operatorname{Ann}(U) . x$ induces an isomorphism of $U / Q B$ onto $Q B$. But this is impossible. Therefore, we have $Q A=Q B$ and hence $A=B$. We now show $C(B)=B$. If not, take $x$ in $C(B)-B$ with $x B$ in $Z(G / B) \cap C(B) / B$. Then $D=\langle x, B\rangle$ is an abelian normal subgroup contradicting the maximality of $A=B$. Since $F$ is not algebraic over a finite field, there exists a monomorphism of $B$ into the multiplicative group of $F$. This defines an $F[B]$ module structure on $F$. We denote this module by $V$. Let $P=\operatorname{Ann}_{F[B]} V . \bar{V}=V \otimes_{F[B]} F[G]$ is an irreducible $F[G]$ module. This follows since if $g_{1}$ and $g_{2}$ are in different cosets of $B$, then $V g_{1}$ and $V g_{2}$ are not isomorphic as $F[B]$ modules since $C[B]=B$. This implies $g_{i} P g_{1}^{-1} \neq g_{2} P g_{2}^{-1}$. Annihilator of $\bar{V}$ is $\left(\cap_{g \operatorname{in} G} g P g^{-1}\right) G$. But this is zero by Bergman's theorem [1]. Hence Ann $(\bar{V})$ is a nonmaximal primitive ideal.

The proof can be simplified considerably if $F$ is a large field. More specifically, if the transcendence degree $F$ is larger than the rank of $G$, a 
theorem of Passman may be applied to produce lots of primitive ideals easily [3].

\section{REFERENCES}

1. G. M. Bergman, The logarithmic limit-set of an algebraic variety, Trans. Amer. Math. Soc. 157 (1971), 459-469. MR 43 \#6209.

2. P. Hall, On the finiteness of certain soluble groups, Proc. London Math. Soc. (3) 9 (1959), 595-622. MR 22 \# 1618.

3. D. S. Passman, Primitive group rings, Pacific J. Math. 47 (1973), 499-506.

4. J. E. Roseblade, The integral group rings of hypercentral groups, Bull. London Math. Soc. 3 (1971), 351-355. MR 45 \# 408.

5. - Group rings of polycyclic groups, J. Pure Appl. Algebra 3 (1973), 307-328. MR 48 \#11269.

6. A. E. Zalesskiī, Irreducible representations of finitely generated nilpotent torsion free groups, Mat. Zametki 9 (1971), 199-210 = Math. Notes 9 (1971), 117-123. MR 46 \#1912.

Department of Mathematics, Virginia Polytechnic Institute and State University, BLACKSBURG, VIRGINIA 24061 